\title{
ON THE DIMENSION OF PARACOMPACT HAUSDORFF SPACES
}

\author{
KEIÔ NAGAMI
}

This short note gives the generalized sum theorem for Lebesgue dimension of paracompact Hausdorff spaces. Our theorem, though it is a generalization of Mr. Morita's sum theorem for fully normal spaces [3, Theorem 3.2] which is essentially based on his generalized sum theorem for normal spaces [3, Theorem 3.1], is obtained by very brief arguments, using only the usual sum theorem for normal spaces.

THeOREM 1. A paracompact Hausdorff space $R$ is $\leqq n$-dimensional if and only if it is locally $\leqq n$-dimensional. ${ }^{1)}$

Proof. 'Only if' part is obvious. We shall prove 'if' part. For every point $p \in R$, there exists an open neighbourhood $V(p)$ whose closure is $\leqq n$ dimensional. Since $R$ is strongly screenable [4, Theorem 2], there exists an open covering $\left\{U_{\alpha} ; \alpha \in \bigcup_{i=1}^{\infty} A_{i}\right\}$ which refines $\{V(p) ; p \in R\}$ such that each $\mathfrak{l}_{i}$ $=\left\{U_{\alpha} ; \alpha \in A_{i}\right\}$ forms a discrete collection. It can easily be seen, from $\leqq n$-dimensionality of $\bar{U}_{\alpha}$ and from discreteness of $\mathfrak{H}_{i}$, that each $\bar{U}_{i}=\bigcup_{\alpha \in A_{i}} \bar{U}_{x}$ is $\leqq n$-dimensional. Thus $R$ is $\leqq n$-dimensional as the sum of countable $\leqq n$-dimensional closed sets [1, Theorem 4.2]. Q.E.D.

Theorem 2. (Generalized sum theorem) If a paracompast Hausdarff space $R$ is covered by a collection of $\leqq n$-dimensional closed sets, $\left\{F_{\alpha} ; \alpha \in A\right\}$, which is locally countable, $R$ is $\leqq n$-dimensional.

Proof. Let $p$ be an arbitrary point of $R$ and $V(p)$ be a neighbourinood of it such that $A(p)=\left\{\alpha ; \overline{V(p)} \cap F_{x} \neq \phi\right\}$ consists of at most countable sets of indices. Since $R$ is normal, $\overline{V(p)}$ is normal as a relative space. Then the sum

Received February 24, 1954.

1) A space is called locally $\leqq n$-dimensional, if every point of the space has a closed neighbourhood whose dimension is at most $n$.

2) A collection of subsets of $R$ is called locally countable, if every point of $R$ has an open neighbourhood of it which meets at most countable elements of the collection. 
theorem for countable closed sets can be applied on it and $V(p)$ is $\leqq n$-dimensional from $\leqq n$-dimensionality of each $V(p) \cap F_{\alpha}$. Q.E.D.

This is a generalization of Mr. Morita's sum theorem for fully normal spaces [3, Theorem 3.2].

COROLlary. Generalized sum theorem holds in every locally compact topological group.

This is a trivial consequence of the above theorem and [2, Theorem 14].

THEOREM 3. Monotonicity of dimension holds in every completely paracompact $^{3)}$ Hausdorff space.

Proof. Let $R$ be such a space which is $\leqq n$-dimensional. To verify monotonicity of dimension, it suffices only to prove $\operatorname{dim} G \leqq n$ for every open subset $G$ of $R$. Since $R$ is regular, $G$ is locally $\leqq n$-dimensional. Hence $G$ is $\leqq n$ dimensional from paracompactness of it. Q.E.D.

Remark. It is well-known that monotonicity of Lebesgue dimension holds in every perfectly normal space. Theorem 3 gives another example such a space that monotonicity of dimension holds in it. It is not so difficult to verify that i) every paracompact Hausdorff space is not always completely paracompact, ii) every metric space is completely paracompact but the converse is not always true, iii) every paracompact, perfectly normal space is completely paracompact. But the author cannot know whether every completely paracompact Hausdorff space is perfectly normal or not.

\section{BIBLIOGRAPHY}

[1] E. Hemmingsen: Some theorems in dimension theory for normal Hausdorff spaces, Duke Math. J. 13 (1946) 495-504.

[2] K. Morita: Star-finite coverings and the star-finite property, Mathematica Japonicae 1 (1948) 60-68.

[3] K. Morita: On the dimension of normal spaces II, J. Math. Soc. Japan 20 (1950) 16-33.

[4] K. Nagami: Paracompactness and strong screenability, Nagoya Math. J. 8 (1955) 79-102.

3) A space is called completely paracompact, if every subspace is paracompact or equivalently if every open subspace is paracompact. 